\title{
IMPLEMENTACIÓN DEL SISTEMA ORAL EN LOS JUZGADOS LABORALES DEL CIRCUITO JUDICIAL DE BUCARAMANGA (COL)
}

\author{
Carolina Valencia Rey \\ Especialista en Derecho Procesal, Universidad Santo Tomás - Bucaramanga (Col.) Abogada, Universidad Santo \\ Tomás - Bucaramanga (Col); Secretaria Juzgado Quinto Laboral del Circuito de Bucaramanga (Col). \\ E-mail: carolinavalenr@hotmail.com
}

\begin{abstract}
Resumen
La justicia laboral en nuestro país y la preeminencia de la misma al regular los derechos y garantías mínimas de los trabajadores, los cuales son irrenunciables, al igual que temas de raigambre nacional como el sistema general de seguridad social colombiano, han generado del legislador la imperiosa necesidad de contrarrestar los altos niveles de congestión judicial y morosidad del Sistema; para tal fin se expidió la ley 1149 de 2007 mediante la cual se ordenó la aplicación del procedimiento oral a los procesos laborales, en todas las etapas procesales. Este trabajo confecciona un análisis práctico de las causas que le dieron origen a la oralidad laboral en nuestro país, los principios procesales sobre las cuales se cimentó la ley en comento y, se desarrolla un sondeo sobre los beneficios obtenidos de los Juzgados Laborales Pilotos de Oralidad en el Distrito Judicial de Bucaramanga.
\end{abstract}

Palabras Clave: Oralidad en el régimen procesal del trabajo, principios procesales de Inmediación y Celeridad; Plan Piloto de la Oralidad.

\begin{abstract}
Labor justice in our country and the preeminence of the same on regulate the rights and minimal guarantees of the workers, which that can not be waived, as topics of national root as the general system of social safety in Colombia, they have generated at the legislator the imperious need to offset the high levels of congestion and sluggishness of the judicial system; for this purpose was issued the law 1149 of 2007, through which ordered the application of the oral procedure to the labor processes, in all the procedural stages. This work makes a practical analysis of the reasons that gave to him origin to the labor orality in our country, the procedural principles on which cemented the law in question, and develops a survey on the benefits of the Labor Judges Pilots of Orality in Bucaramanga's Judicial District
\end{abstract}

Key Words: Orality in the Procedural Regime of the Work, principles of inmediacy and speed, and Pilot Plan of the Orality.

\section{Résumé}

La justice du travail dans notre pays et la règle de celui-ci à réglementer les droits et garanties minimales de travailleurs, qui sont indispensables, ainsi que les questions de racines nationales que le système général de sécurité sociale en Colombie, ont conduit le législateur à l'impératif nécessité de lutter contre les niveaux élevés de congestion et du système judiciaire la délinquance, dans le but de la délivrance de la loi 1149 de 2007 en ordonnant l'application de la procédure orale à des processus de travail dans tous les stades de la procédure. Ce document dresse une analyse pratique des causes qui ont donné lieu à la tradition orale dans notre travail dans les pays, les principes de procédure sur laquelle cimentés la loi en question et développe une enquête sur les avantages de tribunaux du travail des pilotes Oralité district judiciaire de Bucaramanga.

Mots-clés : L'oralité dans le système de contentieux du travail, des principes de procédure de l'immédiateté et Hâte, Plan pilote oralité. 

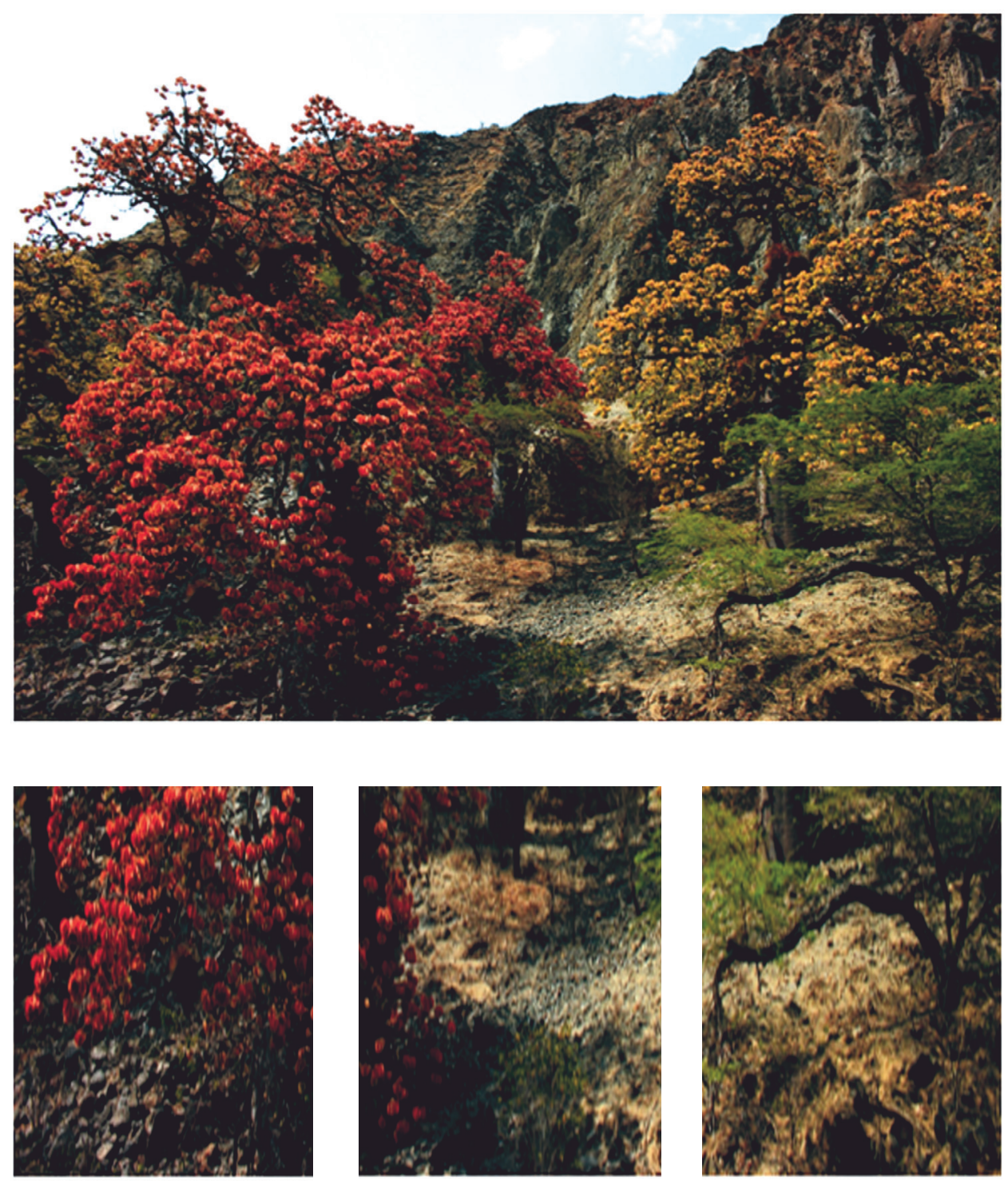

Cañón del Chicamocha - Ceibas Barrigonas

Jorge William Sánchez Latorre 


\section{IMPLEMENTACIÓN DEL SISTEMA ORAL EN LOS JUZGADOS LABORALES DEL CIRCUITO JUDICIAL DE BUCARAMANGA (COL)*}

Carolina Valencia Rey

\section{LA REALIDAD DEL PROCESO LABORAL EN EL ORDENAMIENTO JURÍDICO COLOMBIANO}

Desde el año 2004 en razón de la ley 906, entró en vigencia el Sistema Penal Acusatorio, mediante la demandada implementación de la oralidad en materia penal. En el Distrito Judicial de Bucaramanga el Sistema Penal Acusatorio se acoge a partir del primero $1^{\circ}$ de enero de 2006.

La acción democrática protectora de garantías fundamentales mediante audiencias públicas y la reducción en los tiempos y costos del proceso son algunos de los substanciales frutos arrojados en dicho sistema, lo que estimuló al legislador a decidir que ésta no era la única rama en la justicia colombiana que sería pulsada por la oralidad. Por eso, mediante el Decreto 1098 de 2005, prorrogado posteriormente por los Decretos Nacionales 3640 de 2005 y 3240 de 2006 se creó la Comisión Intersectorial encargada de coordinar, orientar y recomendar las normas, medidas y herramientas necesarias para poner en práctica el principio de oralidad en el Régimen Procesal del Trabajo y de la Seguridad Social en todo el territorio nacional.

Se estimó pertinente la adopción del sistema oral por la necesidad social de generar mayor celeridad en el desarrollo de la relación procesal laboral y de la seguridad social en consonancia con la recta administración judicial, y como resultado de estas exigencias, se expidió la ley 1149 de 2007 mediante la cual se ordenó la aplicación del procedimiento oral a los procesos laborales, en todas las etapas procesales.

El Consejo Superior de la Judicatura, en ejercicio de sus facultades constitucionales y legales acordó ciertas medidas tendientes a poner en operación los Juzgados Laborales del Plan Piloto de la Oralidad; y acorde con esto, en el Distrito Judicial de

* El artículo presenta los resultados sobre la implementación del sistema oral en los juzgados laborales, a partir de la revisión hecha tanto en Juzgados Laborales como en Pilotos de Oralidad. Se deriva del ejercicio de investigación desarrollado por la autora, en el marco de la Asignatura Metodología de la Investigación (Asesores, Javier Díaz Díaz, Elva Santamaría Sánchez) de la Especialización en Derecho Procesal (Cohorte IV). Posgrados en Derecho: Universidad Santo Tomás- Bucaramanga (Col). 
Bucaramanga se encuentran desde agosto de 2009 seis (6) Juzgados Laborales del Circuito, dentro de los cuales tres (3) son Piloto de la Oralidad en la actualidad y los otros tres (3) operan bajo el sistema laboral primigenio.

Ahora bien, la aplicación de dos sistemas simultáneamente ha generado una inseguridad jurídica en la justicia laboral, lo que llevó a pensar si esta mixtura de normas repercutió negativamente en la administración judicial al fomentar y reincidir en las causas estructurales de la congestión y la morosidad de los operadores de justicia, por lo que se requiere juzgados de descongestión en el sistema antiguo, ó analizaremos si por el contrario, es imperiosa la implementación de la oralidad plena en el Distrito Judicial de Bucaramanga en materia laboral, toda vez que la oralidad beneficia principios procesales de inmediación y celeridad de raigambre constitucional, beneficios que se encuentran claramente reflejados en los resultados de los juzgados pilotos de oralidad desde su iniciación en agosto de 2009 hasta diciembre de 2011.

Para analizar adecuadamente estas situaciones, se realiza un análisis claro de los factores que llevaron a la aparición de la oralidad en materia laboral y el resultado de los mismos concretamente en el Distrito de Bucaramanga. En consonancia con lo anterior, se desarrollan tres acápites puntuales, el primero de estos se encuentra destinado a examinar los fundamentos de orden legal y los principios rectores de la oralidad en materia laboral que acarrearon el impulso a hacer efectiva la aplicación del principio de oralidad en el Régimen Procesal del Trabajo y de la Seguridad Social.

En el segundo acápite se presentan las causas que dieron origen a la Oralidad en laboral en Colombia, particularmente desde tres ópticas críticas, las causas sociológicas, nacionales e internacionales. Y finalmente, como parte del tercer acápite y culminación del escrito se presentan los resultados obtenidos por parte de los Juzgados Laborales Piloto de Oralidad en el Distrito Judicial de Bucaramanga durante la vigencia judicial del año 2010 y 2011.

\section{Fundamentos de la oralidad}

Desde la perspectiva jurídica, se ha por decantar que la oralidad no se encuentra acogida en nuestro sistema colombiano como una transposición de las grandes tendencias jurídicas de occidente. Es de percatar que, en la Convención Americana sobre Derechos Humanos -Pacto de San José de Costa Rica- (Aprobada en la Conferencia de los Estados Americanos, en San José el 22 de noviembre de 1969) se establece implícitamente la oralidad, al disponer en su artículo 8.2.f. que durante el proceso toda persona tiene derecho, en plena igualdad y entre otras, a la siguiente garantía mínima:

\footnotetext{
"f) derecho de la defensa de interrogar a los testigos presentes en el tribunal y de obtener la comparecencia, como testigos o peritos, de otras personas que puedan arrojar luz sobre los hechos";
} 
es decir, un debate que ha de ventilarse por el medio oral. Sin embargo, aún bajo los antecedentes históricos, y las connotaciones de índole mundial antes descritas, en Colombia ha predominado por manejar un sistema judicial basado en la escritura, legislación que tiene influencia de aquellos sistemas procesales del continente europeo, como los llamados civil law y common law, que se caracterizan por aquel predominio de la escritura; $y$, frente a lo cual, no es de secreto en el ordenamiento jurídico colombiano, ha conllevado al surgimiento de una excesiva lentitud en la solución de los conflictos y situaciones entre las partes y en torno los terceros que comparecen al proceso en busca de aquella anhelada efectividad de los derechos sustanciales.

En materia laboral fenómenos analizados por la Comisión Intersectorial para la Efectividad del Principio de Oralidad en el Régimen Procesal del Trabajo y de la Seguridad Social como el desempleo, el despido injustificado, la liquidación de empresas, y el irrespeto a derechos de personas en situación de vulnerabilidad propició la congestión en los despachos judiciales laborales colombianos. Razones por las cuales, mediante la ley 1149 de 2007, propuso el legislador satisfacer una demanda de justicia para el trabajador y para los usuarios del servicio público en salud y pensiones, sin dilación puesto que toda postergación significa un alto costo social, con medidas que aseguraran no sólo el efectivo acceso a la administración de justicia, sino el que tengan solución pronta a sus conflictos, como lo exige la naturaleza de sus derechos sociales, con obras y actividades tendientes a hacer operativo el marco normativo procesal vigente, introduciéndole algunas reformas puntuales a la justicia laboral colombiana.

A consecuencia de los anteriores acontecimientos sociales, el análisis exhaustivo del Gobierno decantado mediante la Comisión Intersectorial para la Efectividad del Principio de Oralidad en el Régimen Procesal del Trabajo y de la Seguridad Social indicó que al ser el artículo 53 de la Constitución Política de Colombia, la fuente de los principios del Derecho del Trabajo, y norma

"que tiene valor constitucional, es decir, el más alto dentro de la jerarquía de las fuente en derecho en el país" (Iván Daniel Jaramillo Jassir, Principios constitucionales y legales del derecho del trabajo colombiano. Universidad del Rosario, 2010, p.31)

y conforme a pautas constitucionales, es el propio constituyente quien faculta a nuestros legisladores a expedir el Estatuto del Trabajo que vela por las relaciones laborales cimentado en los principios del Estado Social de Derecho. Puntualiza el doctrinante Iván Daniel Jaramillo Jassir que han pasado casi veinte años y, a pesar de diversas iniciativas parlamentarias para cumplir el mandato constitucional, no se ha expedido el estatuto del trabajo; sin embargo, al momento de la expedición de la ley 1149 de 2007, que transformó la visión del derecho laboral el legislador determinó los criterios y principios que orienten la oralidad en el campo laboral y de seguridad social, parámetros delimitados por la Comisión Intersectorial. 


\section{Principios rectores de la oralidad en materia laboral}

Aquellos lineamientos que estimó el legislador y la Comisión Intersectorial para la Efectividad del Principio de Oralidad en el Régimen Procesal del Trabajo y de la Seguridad Social, que fueron la plataforma estructural por la cual se impulsó en Colombia la cultura de la oralidad como aquel camino magistral para que el Sistema Judicial realizara una eficiente actuación en la justicia laboral y de seguridad social se pueden sintetizar bajo las siguientes medidas.

Es claro que, como premisa mayor, se puede dar que en Colombia el Estado goza del monopolio del poder jurisdiccional - Obligatoriedad de la Jurisdicción-, con las salvedades que la Constitución y la ley han señalado, fundamento de orden constitucional y debidamente acogido por los administradores de justicia, entre tanto se ha señalado que:

“(...) el propósito fundamental de la administración de justicia es hacer realidad los principios y valores que inspiran al Estado Social de Derecho" (Corte Constitucional. Sentencia No. C-037. Magistrado ponente: Dr. Vladimiro Naranjo Mesa. (Febrero, 1996).

$\mathrm{Y}$ en el escenario laboral, esta proposición no es diferencial, dado que no se instituyó como una simple transición escritura-oralidad sino, por el contrario, como una cultura jurídica la cual arroja como resultado un juez administrador de justica pública con presencia constante de las decisiones trascendentales de la litis y, por lo tanto, líder y garante de los derechos de las partes.

Este primer principio, la obligatoriedad de la jurisdicción, se encuentra estrechamente vinculado, con un segundo mandato de estirpe constitucional, la prevalencia del derecho sustancial sobre el procesal acogido por el artículo 228 de la Constitución que establece que en las actuaciones de la Administración de Justicia "prevalecerá el derecho sustancial", para tal aspecto, hacia la realización de los derechos y la solución de los conflictos, el derecho procesal laboral se entiende a nivel jurídico como un medio, dado que, la legislación colombiana ha establecido diversas cláusulas de protección en su código sustantivo mediante las cuales equilibran aquellas relaciones que surgen entre empleados y empleadores.

Tacha con la afirmación anterior, el reciente postulado interpretativo de la Sala Plena de la Corte Constitucional (Corte Constitucional. Sala Plena. Sentencia C-868. Magistrada ponente: Dra. María Victoria Calle Correa (Noviembre, 2010) que puntualiza que el derecho procesal laboral no es un medio sencillamente procedimental, puesto que se han señalado concretas cláusulas de exclusión, distintamente en el articulado del Código Procesal del Trabajo y de la Seguridad Social, a fin de corregir y depurar aquellas actuaciones dirigidas al desmedro de los intereses superiores y del orden público laboral, privilegiadas en nuestra Constitución colombiana y en diversos convenios internacionales. 
Estos derechos, indistintamente de su naturaleza, sustanciales o procesales, se encuentran amparados por el debido proceso, no solo consagrado en la Constitución Colombiana, sino en otros cuerpos normativos de carácter internacional, tales como la Declaración Universal de Derechos Humanos de 1948, en la Convención Americana sobre Derechos Humanos (Pacto de San José de Costa Rica), la Declaración Americana de los Derechos y Deberes del Hombre entendido como

"un conjunto de principios, tales como el de legalidad, el del juez natural, el de favorabilidad, el de presunción de inocencia y el derecho de defensa, los cuales constituyen verdaderos derechos fundamentales" (Corte Constitucional, Sentencia T-1263 del 29 de noviembre de 2001).

Y el cual, un Juez Laboral no puede desatender al momento de conducir por el camino judicial un conflicto suscitado entre un trabajador y un empleador; o en aquellas situaciones jurídicas en las cuales se ventilan derechos pensionales y situaciones que afectan el mínimo vital y móvil de cualquier ciudadano.

Actuaciones que instintivamente, conllevan al análisis de un cuarto principio rector, legalidad, y que en virtud del mismo, se ha aprehendido que las conductas sancionables no sólo deben estar descritas en norma previa sino que, además, deben tener un fundamento legal, por lo cual su definición no puede ser delegada en la autoridad administrativa (Corte Constitucional. Sentencia C-597. Magistrado Ponente: Dr. Alejandro Martínez Caballero (Noviembre, 1996).

Regla judicial y jurídica que se complementa de forma armónica en el Estatuto Laboral y de Seguridad Social colombiano, al entender el derecho laboral como aquel componente mediante el cual el Estado asegura el cumplimiento de aquellas prerrogativas esenciales para el desarrollo y fomento de la comunidad en general e involucra, el constitucionalmente establecido interés colectivo, instante en el cual se fusiona de manera sistémica la legalidad en las actuaciones orales laborales y de seguridad social.

Dichos procesos que por su alcance constitucional se encuentran sumergidos por el principio rector de la celeridad, el cual de raigambre constitucional preside la función judicial, al exigir que las diligencias judiciales se adelanten "sin dilaciones injustificadas" conforme lo dispone el artículo de la Carta Magna. Para tal aspecto, la ley Estatutaria de la Administración de Justicia (Ley 270 de 1996. Reformada por la Ley 1285 de 2009. Enero 22 de 2009. Diario Oficial No. 47.240) establece en su artículo $4^{\circ}$ que:

"la administración de justicia debe ser pronta, cumplida y eficaz en la solución de fondo de los asuntos que se sometan a su conocimiento".

Es la celeridad uno de los pilares propulsores de la implementación de la oralidad en el proceso laboral, puesto que, al modificarse la estructura procesal de la legislación laboral mediante la ley 1149 de 2007, se promovió en materia judicial la capacidad de responder efectivamente a las necesidades e intereses reales del contexto local y regional, acorde con las políticas nacionales a fin de obtener por parte del poder 
jurisdiccional la recuperación e incremento de la sensibilización y la humanización del proceso al fortalecer el respeto por los derechos en los términos del Estado Social de Derecho.

El referido principio se encuentra conexo con los principios de publicidad $y$ gratuidad, entendidos como presupuestos de la democracia participativa colombiana, al establecer el Constituyente en el Artículo $2^{\circ}$ la publicidad como el

"reconocimiento del derecho que tiene la comunidad a conocer las actuaciones de las autoridades públicas y, a través de ese conocimiento, a exigir que ellas se surtan con total sometimiento a la ley" (Corte Constitucional. Sentencia C-1114. Magistrado Ponente: Dr. Jaime Córdoba Triviño, Noviembre, 2003).

El proceso laboral, no se aparta de este precepto al asumir la publicidad como una garantía de transparencia en la actuaciones mediante las cuales se resuelven circunstancias que atañen relaciones empleador-trabajador, y de seguridad social.

En el marco de estos principios, se define en el Código Sustantivo del Trabajo que rige en Colombia desde hace más de 54 años, el principio de gratuidad en el procedimiento laboral, como el conducto mediante el cual se equilibran las balanzas de justicia social estipuladas en nuestra Carta Magna y de equidad en Convenios Internacionales; en el entendido que el Constituyente estimó al trabajo como un valor y un derecho fundamental, por lo que se necesitaba imperiosamente proteger la parte más débil de la relación laboral que en este caso incumbe al trabajador, entendiéndose, claro está, que toda regla general posee excepciones las cuales se encuentran relacionadas de manera precisa y clara en la ley.

Finalmente, los postreros pero no menos importantes principios rectores de la oralidad en materia laboral son la inmediación y concentración. El juez como director dinámico del proceso, exige de tal calidad un control auténtico el cual no se lograba en materia laboral mediante el sistema escrito por la delegación en los subalternos y la atomización en varias audiencias, y el cual ha de lograr su umbral mediante una relación directa por parte del dispensador de justicia con los sujetos procesales y con los materiales elementos de convicción mediante la concentración e inmediación plena del administrador de justicia con la litis definida, en conexidad con la concentración de la prueba dentro del mismo proceso, y de las audiencias como tal, las cuales fueron concentradas y reducidas en su numerosidad.

Son los anteriores principios sucintamente analizados por los cuales el legislador inspiró la transformación netamente procesal en materia laboral y de seguridad social en Colombia reflejada en la ley 1149 de 2007; sin embargo: ¿Era totalmente imprescindible la expedición de esta normativa?

Para dar respuesta a este interrogante, se ocupará en el siguiente capítulo de analizar sucintamente las causas originarias del fenómeno de la oralidad en materia laboral en Colombia, para así al tener claro los principios y las causas de la ley 1149 de 2007 poder establecer, a partir de los resultados obtenidos en los Despachos Judiciales Pilotos de la Oralidad en Bucaramanga, si la oralidad efectivamente beneficia al proceso laboral y de seguridad social en nuestro país. 


\title{
Causas que dieron origen a la oralidad laboral en Colombia
}

Del epítome previamente labrado en conexidad con los principios relevantes de la oralidad en materia laboral, se ha interpretado que dicha metamorfosis no era imprescindible, tal como lo expuso en su oportunidad el Presidente del Tribunal Superior del Distrito Judicial de Medellín al indicar que:

\begin{abstract}
"grave es una justicia tardía, pero más nefasta la que lesione los derechos de las partes en aras una pretendida celeridad (Comisión Intersectorial. Foro de Medellín, 24 de Junio de 2005. Intervención de Tascón Rico Óscar de Jesús.) si esta rapidez pone en riesgo derechos fundamentales.
\end{abstract}

Por lo que se hace imperioso un examen meticuloso de las causas reales que le dieron origen a la oralidad laboral en Colombia, para poder cotejar las dos posturas, positiva y negativa, frente a la implementación de la oralidad en el proceso laboral, y determinar con precisión, cuál finalmente es la más proteccionista de derechos sustanciales.

\section{Causas Sociológicas}

Como una de las causas que dieron origen y sostienen incólume al sistema oral en los procesos laborales y de seguridad social son los cometidos sociológicos del Estado Social de Derecho, dentro del que se resalta el proporcionar una pronta y cumplida justicia a sus usuarios (Artículos $2^{\circ}$ y 228 de la Constitución Política de Colombia de 1991) y conforme el capítulo precedente, ésta no es una de las cualidades y atributos de los que goza la Rama Judicial en Colombia, lo cual ha decantado en los ciudadanos una falta de creencia y confianza en el sistema judicial, que no genera consecuencias positivas en la Comunidad sino, por el contrario, se contraponen con el principio de la obligatoriedad de la jurisdicción al conllevar al ciudadano a ejercer justicia propia mediante la ley del Talión (lex talionis), una de sus expresiones más famosas es el "ojo por ojo y diente por diente" o acudir a tendencias pendencieras y anarquizantes a fin de obtener sus beneficios individuales, y dejar a un lado la convivencia social, como resultado de la mentalidad del ciudadano que ha de vincular al sistema judicial como una rama controladora de la paz nacional; y, por ende, estimar que si el Estado no es capaz de asegurar una paz interna mediante los mecanismos judiciales establecidos, es viable que se estime acudir a entes subversivos al margen de la ley a fin que sean ellos los encargados de impartir justicia; para dejar por completo a un lado el ordenamiento jurídico legítimo.

Como consecuencia del fenómeno que se ha descrito, al no reconocerse la capacidad de administrar justicia por parte del Estado, se enlaza por consecuencia lógica la desfiguración del juez como operador y dispensador de justicia, dado que su actuar se ve inmerso de morosidad y congestión. Y sin extralimitarse a las actuaciones ilegales, se puede corroborar lo anterior en el reconocimiento y la capacidad de otros sujetos de producir y aplicar derecho mediante la existencia de diversas formas de justicia no judiciales, y ni siquiera estatales, como consecuencia de un pluralismo jurídico. 
Son las anteriores, algunas de las innumerables razones por las cuales mediante la oralidad acorde con la inmediación y la celeridad propende el legislador en recobrar la credibilidad en el Estado y concretamente en el sistema de justicia, y de esta forma generar que la sociedad sea partícipe de las actuaciones judiciales, que conlleva a una conciencia social exhortada de la existencia de una recta y ampliada administración de justicia, y restablecer así la confianza en las instituciones judiciales, y concretamente en el juez laboral.

\section{Causas Nacionales}

Dentro de los factores que estimó la Comisión ${ }^{1}$ como causales de congestión y atraso en materia laboral, se destacaron: la inestabilidad económica del país, el crecimiento y concentración de la población urbana, la quiebra y liquidación de empresas y entidades del gobierno, la reestructuración del Estado, y el egreso de procesos similares a la demanda, lo que impide la realización de acciones de disminución del inventario actual; última característica visible notoriamente acorde con el siguiente cuadro expuesto en el 2005 por la Comisión:

Tabla 1. Inventario Final de expedientes obrantes en los Tribunales Superiores Sala Laboral de Colombia desde 2000 a 2004

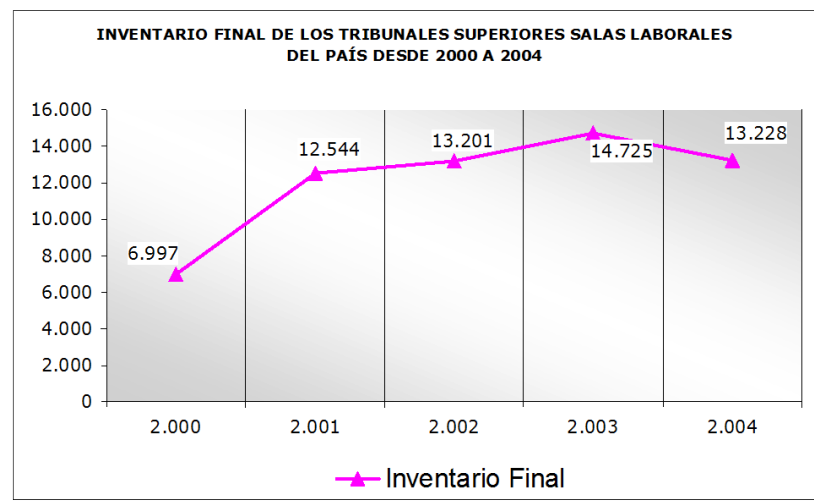

Fuente: (Plan de Acción Para la Efectividad de la Oralidad en el Régimen Procesal del Trabajo y de la Seguridad Social. Congestión y atraso histórico en el área laboral. Unidad de Desarrollo y Análisis Estadístico, 2005, 50).

Para la comunidad jurídica laboral las directrices trazadas por los redactores del Código Procesal Laboral de 1948 fueron adecuadas, pero en la actualidad se requerían de acciones para ajustar la jurisdicción laboral y de la seguridad social a

1 Informe de los invitados permanentes de la Comisión Intersectorial para la Efectividad del Principio de Oralidad en el Régimen Procesal del Trabajo y de la Seguridad Social (Septiembre, 2005). 
las demandas de la sociedad de hoy en día y, de esa manera, satisfacer los ruegos de justicia del trabajador y de los usuarios del servicio públicos en salud y pensiones, sin dilaciones.

Por lo tanto, se estimó por parte de la Comisión, de la adopción de medidas integrales, mediante:

1. La adecuación de la estructura judicial - distribución, número y organización de despachos judiciales, de Magistrados y de Jueces, suficiente y adecuada.

2. El equipamiento de los Despachos de espacios y medios tecnológicos necesarios para administrar justicia eficientemente.

3. Adecuación normativa, puntual de manera inmediata, y una revisión extensa a la normatividad procesal laboral y civil a la que aquélla se remite.

4. Capacitación vigorosa y con cobertura total a jueces, magistrados, empleados judiciales y abogados. (Capítulo presupuestos para la efectividad de la oralidad. Plan de Acción para la Efectividad de la Oralidad en el Régimen Procesal del Trabajo y de la Seguridad Social. Septiembre de 2005).

\section{Causas Internacionales}

Los convenios y Tratados Internacionales del trabajo debidamente ratificados por Colombia son parte del ordenamiento jurídico conforme se estipula en la Constitución Política en su artículo 53 y el inciso segundo del artículo 93; sin embargo, ha estimado la Corte Constitucional que los convenios internaciones del trabajo ratificados por Colombia no pueden ser considerados como parámetros supletorios en el ordenamiento laboral y adicionalmente aclara que los convenios que integran el bloque de constitucionalidad en sentido lato orientan la interpretación de la norma constitucional (Constitución Política, Art. 93, inc. 2) y los convenios que integran el bloque de constitucionalidad en sentido estricto han de prevalecer en el orden interno (Constitución Política, Art. 93, inc. 1) (Corte Constitucional. Sentencia C - 401. Magistrado Ponente: Dr. Manuel José Cepeda Espinosa, Abril, 2005).

La Comisión de Aplicación de Normas de la Conferencia, quien actúa como mecanismo esencial del sistema de control de la Organización Internacional del Trabajo ha efectuado desde 1985 un seguimiento constante a Colombia, concretamente por las diver sas quejas y denuncias recibidas por las Organizaciones Internacionales respecto a la violación de derechos humanos de sindicalistas y a la divergencias legislativas entre el ordenamiento jurídico colombiano y los respectivos Convenios de la OIT ratificados por Colombia. Por lo cual, el gobierno colombiano se ha debido presentar en $18^{2}$ oportunidades ante las Comisiones respectivas de los entes internacionales a rendir informes de la situación laboral en Colombia, y ha sido por estas constantes insistencias internacionales que Colombia ha logrado en materia legislativa un gran avance en legislación sindical.

2 http://www.derechoshumanos.gov.co/prensa/destacados... enviado por mail. 
Recientemente, la Organización Internacional del Trabajo, mediante El Panorama Laboral 2010 (Oficina Regional para América Latina y el Caribe. Primera Edición. 2010), incluye en resultados de su Observatorio de la Coyuntura un balance de los alcances de la crisis y recuperación sobre el empleo informal para 5 países (Colombia, Ecuador, México, Panamá y Perú), que incluye algunos indicadores de incidencia de la informalidad por país, los cuales se presentan en el QUIPUSTAT (Sistema de Información Laboral para América Latina y el Caribe), y se resalta que las condiciones laborales se han traducido en varios países en una creciente caída, aunque sus estadísticas enuncian un aumento de la demanda laboral. Y, concretamente, dentro de sus cuadros estadísticos Colombia se encuentra dentro del segundo país con más desempleo urbano, conforme el siguiente Gráfico estadístico:

Figura 1: Tasa desempleo urbano en América Latina

\section{AMÉRICA LATINA Y EL CARIBE (PAISSES SELECCIONADOS): TASA DE DESEMPLEO URBANO, ENERO-SEPTIEMBRE DE $2009 \mathrm{Y}$ ENERO-SEPTIEMBRE DE 2010。 \\ (En porcentajes)}

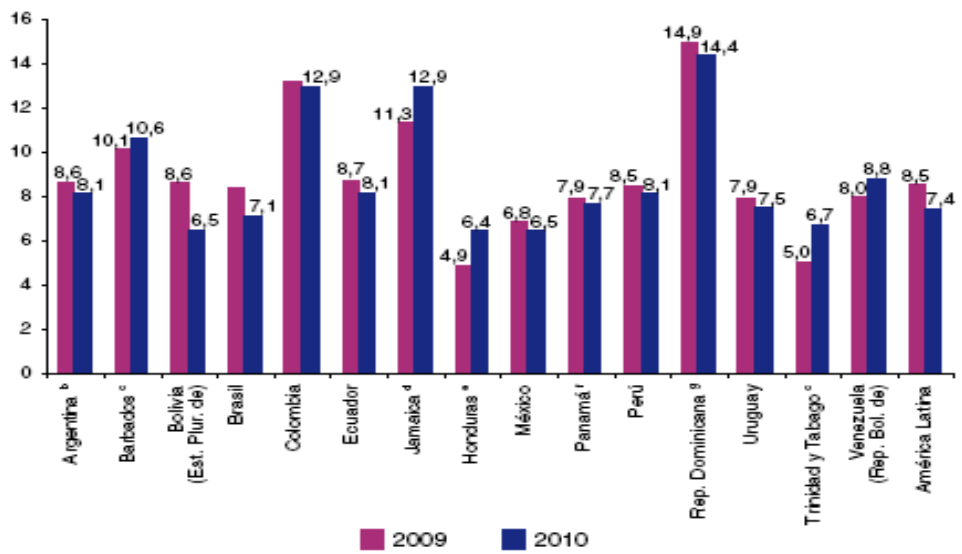

Fuente: Comisión Económica para América Latina y el Caribe (CEPAL) y Organizaciór Internacional del Trabajo (OIT), sobre la base de información de las encuestas de hogares de los países.

Aunque Colombia no fue seleccionada recientemente en estos informes, toda vez que la Comisión de Expertos destacó al Gobierno y a la Nación como un caso en proceso, según el Informe de la Comisión de Expertos en Aplicación de Convenios y Recomendaciones de $\mathrm{OIT}^{3}$, sus estadísticas reflejan sendas irregularidades en materia laboral en el país.

3 Recuperado de :http://www.ilo.org/wcmsp5/groups/public/@ed_norm/@normes/documents/ publication/wcms_145221.pdf actualizado a septiembre de 2010. 
Las cuales conllevan no solamente a índices de desempleo, sino a la concatenación de circunstancias jurídicas que desembocan finalmente en acciones judiciales que han de ser sometidas al control jurisdiccional por parte de los jueces laborales y de seguridad social. Dentro de las que cabe resaltar, las faltas de garantías laborales en cuanto al no pago de aquellos derechos irrenunciables de los trabajadores, sucesos y eventos de despidos injustificados, las respectivas liquidaciones de empresas con porcentaje estatal que conllevan a despidos masivos entre otros; actuaciones que de forma inmersa involucran al poder judicial, al ser esté el garante último de los derechos por las anteriores acciones violatorias.

Aunque los avances logrados por Colombia han sido importantes, para las Organizaciones Internaciones en asuntos laborales ello no es suficiente, y procuran mediante sus recomendaciones que Colombia efectúe un desarrollo legislativo nacional enlazado con un diseño de estrategias y de políticas nacionales cuyo objetivo final sea generar trabajo decente y se complemente con ello las normas internacionales que la OIT ha elaborado en los campos del empleo, las condiciones de trabajo, la política salarial y de remuneración, la seguridad y la salud en el trabajo y la protección social.

Bajo estos argumentos, dentro del Plan de acción para la efectividad de la Oralidad en el Régimen Procesal del Trabajo y de la Seguridad Social efectuado por la Comisión Intersectorial para la efectividad del Principio de Oralidad en el Régimen Procesal del Trabajo y la Seguridad Social, tomo estas comisiones internacionales como una de las causas por las cuales la implementación del sistema oral en el proceso laboral generaría una aplicación efectiva de las normas sobre los derechos fundamentales en el trabajo.

Dicho lo anterior, es pertinente precisar cuáles han sido los resultados arrojados por los Juzgados Pilotos de Oralidad, como el reflejo práctico y evolutivo de los beneficios que indicó el legislador conllevaría la ley 1149 de 2007, y si estos seriamente se están viendo irradiados en los litigios laborales y de seguridad social.

\section{Juzgados laborales pilotos de la oralidad en Bucaramanga: una lectura a los resultados}

\section{Plan piloto}

Como resultado de la ley 1149 de 2007, por la cual se reforma el Código Procesal del Trabajo y de la Seguridad Social para hacer efectiva la oralidad en sus procesos, conforme las causas que se citaron anteriormente el Consejo Superior de la Judicatura, mediante el Acuerdo PSAA09-6103 del 21 de julio de 2009, emanado de la Sala Administrativa creó, a partir del 3 de agosto de 2009 tres juzgados laborales pilotos de la oralidad en Bucaramanga. 
Se implementa en la actualidad dos sistemas simultáneamente, los Juzgados Primero, Segundo y Cuarto efectúan en sus actuaciones judiciales el método escritural primigenio; y los Juzgados Tercero, Quinto y Sexto una mixtura escrituraoralidad por ser pilotos.

Los resultados de los mismos son ostensiblemente notorios, de conformidad con los cuadros que a continuación se relacionan, producto de la investigación efectuada respecto de los descritos Despachos Judiciales en la vigencia de los años 2010 y 2011:

\section{Procesos Ordinarios Laborales}

\begin{tabular}{|c|c|c|}
\hline \multicolumn{2}{|c|}{ JUZGADO PRIMERO LABORAL DEL CIRCUITO DE BUCARAMANGA* } \\
\hline TRIMESTRE & 2010 & 2011 \\
\hline PRIMER & 44 & 15 \\
\hline SEGUNDO & 20 & 31 \\
\hline TERCERO & 26 & 29 \\
\hline CUARTO & 50 & 19 \\
\hline TOTAL & 140 & 94 \\
\hline
\end{tabular}

Nota*: En el Juzgado Primero Laboral del Circuito de Bucaramanga, el cual aún conserva el pleno sistema escritural, en la vigencia judicial del año 2010 emitió un total de 140 sentencias de procesos ordinarios laborales y en la vigencia judicial del año 2011 un total de 94 sentencias de procesos ordinarios laborales, con un estimado entre la radicación del expediente ante la Oficina Judicial y el pronunciamiento de la sentencia de primera instancia de aproximadamente 17 meses.

\begin{tabular}{|c|c|c|}
\hline \multicolumn{2}{|c|}{ JUZGADO SEGUNDO LABORAL DEL CIRCUITO DE BUCARAMANGA* } \\
\hline TRIMESTRE & 2010 & 2011 \\
\hline PRIMER & 35 & 22 \\
\hline SEGUNDO & 37 & 05 \\
\hline TERCERO & 27 & 11 \\
\hline CUARTO & 23 & 06 \\
\hline TOTAL & 122 & 44 \\
\hline
\end{tabular}

Nota*: En el Juzgado Segundo Laboral del Circuito de Bucaramanga, el cual aún conserva de igual forma el pleno sistema escritural, en la vigencia judicial del año 2010 emitió un total de 122 y en la vigencia judicial del año 2011 un total de 44 sentencias de procesos ordinarios laborales, con un estimado entre la radicación del expediente ante la Oficina Judicial y el pronunciamiento de la sentencia de primera instancia de aproximadamente 20 meses. 


\begin{tabular}{|c|c|c|}
\hline \multicolumn{2}{|c|}{$\begin{array}{c}\text { JUZGADO TERCERO LABORAL DEL CIRCUITO DE BUCARAMANGA- PILOTO } \\
\text { DE LA ORALIDAD* }\end{array}$} \\
\hline TRIMESTRE & 2010 & 2011 \\
\hline PRIMER & 31 & 42 \\
\hline SEGUNDO & 17 & 47 \\
\hline TERCERO & 25 & 55 \\
\hline CUARTO & 28 & 25 \\
\hline TOTAL & 101 & 169 \\
\hline
\end{tabular}

Nota*: En el Juzgado Tercero Laboral del Circuito de Bucaramanga, el cual funge como piloto de la oralidad, en la vigencia judicial del año 2010 emitió un total de 101 sentencias de procesos ordinarios laborales, y en la vigencia del año 2011 profirió un total de 169 sentencias de procesos ordinarios laborales, con un estimado entre la radicación del expediente ante la Oficina Judicial y el pronunciamiento de la sentencia de primera instancia de aproximadamente 05 meses.

\begin{tabular}{|c|c|c|}
\hline \multicolumn{2}{|c|}{ JUZGADO CUARTO LABORAL DEL CIRCUITO DE BUCARAMANGA* } \\
\hline TRIMESTRE & 2010 & 2011 \\
\hline PRIMER & 8 & 42 \\
\hline SEGUNDO & 33 & 47 \\
\hline TERCERO & 36 & 43 \\
\hline CUARTO & 62 & 39 \\
\hline TOTAL & 139 & 171 \\
\hline \hline
\end{tabular}

Nota*: En el Juzgado Cuarto Laboral del Circuito de Bucaramanga, el cual a su vez aún conserva de igual forma el pleno sistema escritural, en la vigencia judicial del año 2010 emitió un total de 139 y en la vigencia del año 2011 profirió un total de 171 sentencias de procesos ordinarios laborales, con un estimado entre la radicación del expediente ante la Oficina Judicial y el pronunciamiento de la sentencia de primera instancia de aproximadamente 24 meses. 


\begin{tabular}{|c|c|c|}
\hline \multicolumn{3}{|c|}{$\begin{array}{l}\text { JUZGADO QUINTO LABORAL DEL CIRCUITO DE BUCARAMANGA } \\
\text { PILOTO DE LA ORALIDAD* }\end{array}$} \\
\hline TRIMESTRE & 2010 & 2011 \\
\hline PRIMER & 15 & 35 \\
\hline SEGUNDO & 21 & 39 \\
\hline TERCERO & 24 & 37 \\
\hline CUARTO & 29 & 18 \\
\hline TOTAL & 89 & 129 \\
\hline
\end{tabular}

Nota*: En el Juzgado Quinto Laboral del Circuito de Bucaramanga, el cual funge como piloto de la oralidad, en la vigencia judicial del año 2010 emitió un total de 89, y en la vigencia del año 2011 profirió un total de 129 sentencias de procesos ordinarios laborales, con un estimado entre la radicación del expediente ante la Oficina Judicial y el pronunciamiento de la sentencia de primera instancia de aproximadamente 05 meses.

\begin{tabular}{|c|c|c|}
\hline \multicolumn{3}{|c|}{$\begin{array}{l}\text { JUZGADO SEXTO LABORAL DEL CIRCUITO DE BUCARAMANGA- } \\
\text { PILOTO DE LA ORALIDAD* }\end{array}$} \\
\hline TRIMESTRE & 2010 & 2011 \\
\hline PRIMER & 26 & 26 \\
\hline SEGUNDO & 28 & 38 \\
\hline TERCERO & 61 & 46 \\
\hline CUARTO & 26 & 23 \\
\hline TOTAL & 141 & 133 \\
\hline
\end{tabular}

Nota*: En el Juzgado Sexto Laboral del Circuito de Bucaramanga, el cual funge como piloto de la oralidad, en la vigencia judicial del año 2010 emitió un total de 141, y en la vigencia del año 2011 profirió un total de 133 sentencias de procesos ordinarios laborales, con un estimado entre la radicación del expediente ante la Oficina Judicial y el pronunciamiento de la sentencia de primera instancia de aproximadamente 06 meses.

Es claro los resultados arrojados por los cuadros anteriores, en los cuales patentemente se detecta que los juzgados pilotos de oralidad en el Distrito Judicial de Bucaramanga han efectuado sus labores de una forma más veloz y ágil a comparación de los Despachos Judiciales que no aplican la oralidad dentro de sus procedimientos. Lapsos de 5 y 6 meses para emitir fallo desde la radicación del expediente, son ostensiblemente inferiores a los meses 24 y 20 que arroja entre otros los despachos escriturales. El siguiente cuadro, sintetiza los cuadros anteriores:

De igual forma, la evacuación de procesos que resuelven mediante sentencia de primera instancia asuntos de índole laboral es mayor en los Juzgados Piloto de 
Promedio de meses entre Radicacióny Sentencia Proceso Ordinario Laboral Años 2010 y 2011

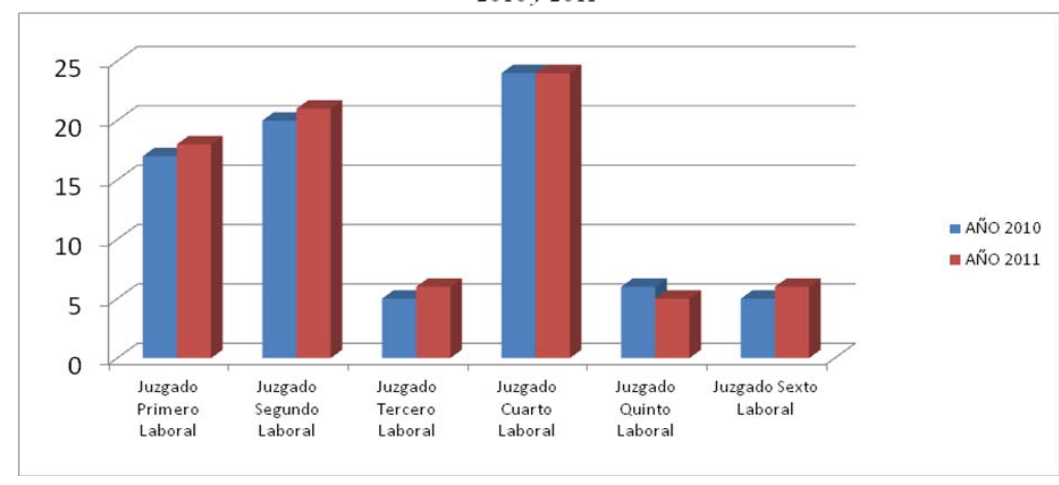

Oralidad que en aquellos que manejan un sistema escritural, esto se refleja en que la totalidad de sentencias proferidas por el Juzgado Primero, Juzgado Segundo y Juzgado Cuarto Laboral se emitieron un total de 710 sentencias judiciales en la vigencia 2010 y 2011. Y en los Juzgados Tercero, Quinto y Sexto Pilotos de Oralidad se profirieron un total de 762 sentencias judiciales en la vigencia 2010 y 2011. Este aspecto, se recalca aun más en la siguiente Gráfica:

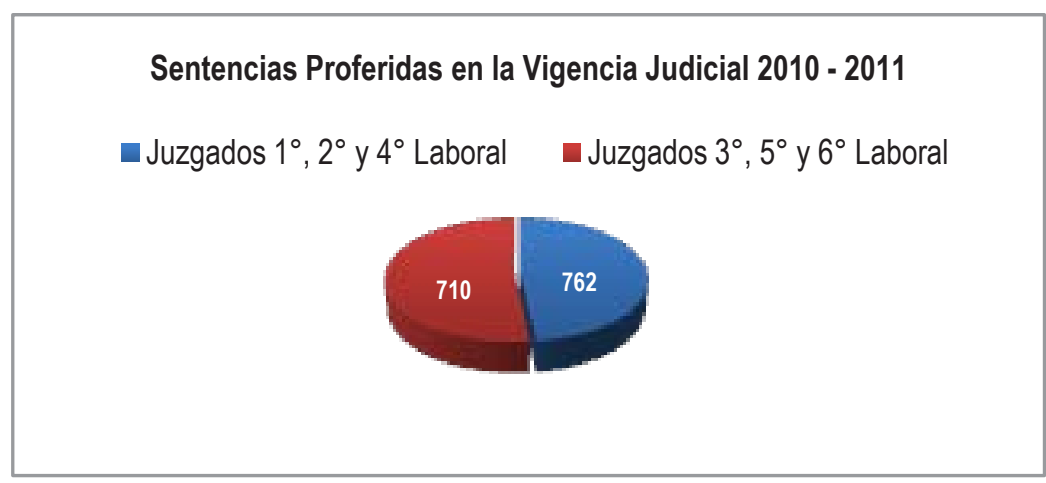




\section{CONCLUSIONES}

La aplicación de dos sistemas simultáneamente ha generado una inseguridad jurídica en la justicia laboral, sin embargo, los litigantes y los dispensadores de justicia en el ámbito laboral dentro de la competencia delimitada por el Distrito Judicial de Bucaramanga, se encuentran en etapa de preparación e instrucción mediante las técnicas desplegadas por los Despachos Pilotos de Oralidad, para dar inicio al cumplimiento de las directrices que imparta el Consejo Superior de la Judicatura, la oralidad plena en este Distrito Judicial.

Se puede apreciar y dilucidar uno de los primeros interrogantes planteados. Que esa mixtura de normas, en momento alguno ha fomentado morosidad judicial, sino por el contrario, ha incidido en la evacuación rápida de procesos laborales y de seguridad social, por cuanto matemáticamente son dos juzgados más los creados, y consecuentemente la distribución ha de ser prorrateada entre los mismos, es de recordar que los despachos quinto y sexto laboral del circuito de Bucaramanga son creados en agosto de 2009.

De los anteriores axiomas, se puede desprender otra conclusión exteriorizada durante el trayecto de la presente investigación; los juzgados de descongestión que se encaminen procesalmente por el método escritural antiguo están conminados al quebranto procesal de la justicia laboral y, por ende, a su fracaso. Por cuanto, aunque los mismos, tengan su razón de ser exclusivamente frente a la etapa de juzgamiento, el sacrificio que el proceso ordinario ha padecido para llegar a su punto cúspide -sentencia ordinaria laboral de primera instancia- repercute negativamente en la morosidad tanto del juzgado de conocimiento como del encargado de emitir el fallo mediante el proceso de descongestión judicial, sacrificio procesal que se palpa claramente si del mismo se conlleva una etapa probatoria superior a los dos (02) años concretamente por las largas distancias ejecutadas por el Despacho para aplazar las audiencias y fijar fechas posteriores, como se reveló de los estimados indicativos en la Figuras y Tablas del presente escrito.

De las estadísticas recientemente reveladas, se colige que la implementación de los Juzgados Laborales del Circuito Pilotos de la Oralidad en el Distrito Judicial de Bucaramanga han beneficiado en sí mismos a los procesos laborales y de seguridad social por cuanto de los mismos se puede extraer sin mayor vacilación una prontitud en la Administración de Justicia, unos términos no sacrificados, sino acortados por el principio de juez director del proceso. Y por cuanto, a través de la Ley 1149 de 2007 ostentosamente se redujeron las etapas principales a tan solo dos audiencias judiciales y a un máximo de 6 a 8 meses de trámite; mientras que en los juzgados que observamos dentro de los cuadros comparativos que no son pilotos de oralidad concurren a cuatro o más audiencias, si el juez lo considera necesario, se incumple con el principio de concentración inspirado por el constituyente, y alarga en meses y años el pronunciamiento judicial mediante la sentencia de primera instancia.

Consonante con lo anterior, y en total acuerdo del planteamiento del maestro Cappelletti (Cappelletti, 1972). Como consecuencia de la presente investigación 
se considera que la oralidad permite una revalorización de la prueba oral ya que considera que este procedimiento rinde sus mejores resultados en la fase probatoria, por la integridad del principio de inmediación que exige la oralidad del mismo sistema judicial.

Como conclusión substancial, ha de ultimarse que es imperioso la implementación de la oralidad plena en el Distrito Judicial de Bucaramanga en materia laboral, toda vez que la oralidad como se evidenció en la presente investigación beneficia los principios procesales de la inmediación, la celeridad y la concentración de raigambres constitucionales y que fusionan los restantes principios analizados en el presente trabajo investigativo, beneficios los cuales se encuentran claramente reflejados en los resultados de los juzgados pilotos de oralidad desde su iniciación y durante la vigencia judicial del año 2010 y 2011.

Con ello se hace necesario, tener presente que el sistema oral cumple los fines del Estado Social de Derecho, de dar solución pronta y ágil, a los sujetos procesales que se encuentran inmersos en relaciones labores y de seguridad social.

\section{REFERENCIAS}

Boletín CEPAL / OIT Coyuntura laboral en América Latina y el Caribe (Diciembre de 2010), en: http://white.oit.org.pe/estad/laclispub/crisis.php\#click07

Cappelletti, (1972). La Oralidad y las Pruebas en el Proceso Civil. Buenos Aires: Ediciones Jurídicas Europa-América)

Código Procesal del Trabajo y la Seguridad Social. Decreto Ley 2158 de 1948. Junio 24 de 1948

Constitución Política de Colombia. Artículo 53. Publicada en la Gaceta Constitucional No. 116 de 20 de julio de 1991

Diccionario Jurídico de Derecho Laboral (Francisco Manuel Sarmiento Cuadros 2001)

Guía teórica y práctica de Derecho Procesal del Trabajo y la Seguridad Social (Gerardo Botero Zuluaga. Editorial Ibáñez 2008)

La Oralidad en el Derecho Procesal Laboral conforme con la ley 1149 de 2007 (Hugo Alexánder Bedoya Díaz. Leyer 2008)

La Oralidad Laboral: Teoría Práctica y Jurisprudencia. Derecho Procesal del Trabajo y de la Seguridad Social (Fabián Vallejo Cabrera, 5 Ed. Librería Jurídica Sánchez R. - 2008)

Ley 712 de 2001. Por la cual se reforma el Código Procesal del Trabajo. 8 de diciembre de 2001. Diario Oficial No. 44.640

Ley 1149 de 2007. Por la cual se reforma el Código Procesal del Trabajo y de la Seguridad Social para hacer efectiva la oralidad en sus procesos. 13 de julio de 2007. Diario Oficial No. 46.688 
O.I.T. (Organización Internacional del Trabajo)

Oficina Internacional del Trabajo. Comisión de Aplicación de Normas de la Conferencia. Recuperado el día 03 del mes de mayo del año 2011, del sitio Web de nombre del sitio URL: http://www.ilo.org/wcmsp5/groups/public/@ ed_norm/@normes/documents/publication/wcms_145221.pdf

Principios constitucionales y legales del derecho del Trabajo Colombiano (Iván Daniel Jaramillo Jassir. Universidad del Rosario 2010)Fuente: Boletín CEPAL / OIT Coyuntura laboral en América Latina y el Caribe (Diciembre de 2010.

Reforma del Código Procesal del Trabajo y de la Seguridad Social (Ernesto Jiménez Díaz. Legis 2007) 